\title{
UMA PROPOSTA PARA DETERMINAR A INFLUÊNCIA DOS DADOS DE POSICIONAMENTO DOS TRENS DE POUSO NO ALINHAMENTO DIRECIONAL DE UMA AERONAVE
}

\author{
GILSON S. GOMES
}

1. Instituto Tecnológico de Aeronáutica

Pós- Graduação em Engenharia Aeronáutica e Mecânica, Sistemas Aeroespaciais e Mecatrônica

\begin{abstract}
This article presents the application of Factorial Design concepts in an experiment in order to verify if landing gear position factors are for final directional alignment of the aircraft.

Keywords_ Landing Gear, Alignment, Aircraft

Resumo- Este artigo apresenta a aplicação dos conceitos de Projetos fatoriais em um experimento [2],afim de verificar se os fatores de posicionamento dos trens de pouso são significantes para o resultado final do alinhamento direcional de uma aeronave.

Palavras-chave— Trem de Pouso, Alinhamento, Aeronave
\end{abstract}

\section{Introdução}

A verificação final do alinhamento direcional de uma aeronave é realizada na fase de voo de produção, avaliando o seu comportamento direcional a baixa velocidade.

Antes deste processo, existe na fase de produção de uma aeronave, a verificação do alinhamento dos componentes estruturais da mesma tal como seção da asa, unidades da empenagem, superfície de comandos, motores e trem de pouso. Esta verificação é feita através de um processo denominado por triangulação [3].

Um dos fatores avaliados neste processo e de interesse deste trabalho é o posicionamento dos trens de pouso no que diz respeito ao alinhamento e assimetria.

Este trabalho tem como base um estudo através de um modelo de simulação foram avaliados os fatores de influencia no alinhamento direcional da aeronave durante o seu taxiamento.

Os fatores de posicionamento dos trens de pouso são obtidos no processo de triangulação conforme segue:

\subsection{Fator: Posicionamento de Trem de Pouso}

Posições de Assimetria: Verificada através de determinado pontos em cada trem de pouso até dois pontos de referência, obtidos através da linha de centro da aeronave.

Posições de Paralelismo: Verificada através de dois pontos no trem de pouso até a linha de centro da aeronave.

\subsection{Fator: Variação de Posicionamento}

Variação das posições citadas, limitadas pela tolerância especificada pelo fabricante da aeronave.

\section{Propósito}

\subsection{Deslocamento lateral da aeronave}

O quanto a variação das posições de paralelismo e assimetria podem gerar de deslocamento lateral da aeronave (isto é deslocamento para esquerda ou direita), durante o seu taxiamento em baixa velocidade.

\section{Métodos}

\subsection{Unidades Experimentais}

No estudo de caso foi considerada uma arquitetura de triangulação, representada de forma simplificada na figura 1
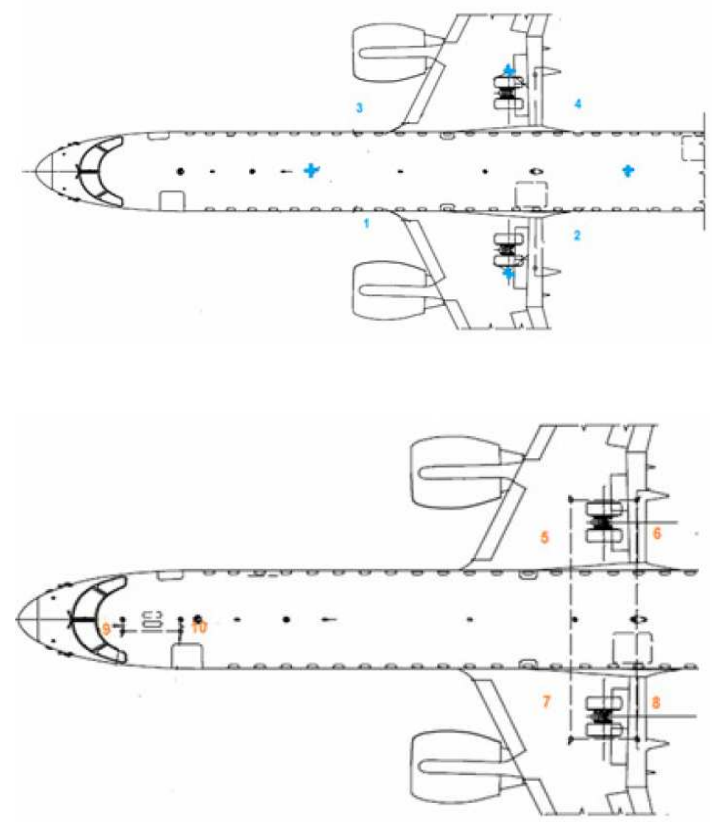

Fig 1 
A unidade dos valores de posição e deslocamento é dada em metros (m).

Valor de tolerância adotado (para efeito de simulação) para a diferença de cada posição: $10 \mathrm{~mm}$

\subsection{0 teste}

No processo de avaliação do alinhamento da aeronave, a mesma é posicionada na linha central da pista sendo avaliada a sua trajetória conforme figura 2 .

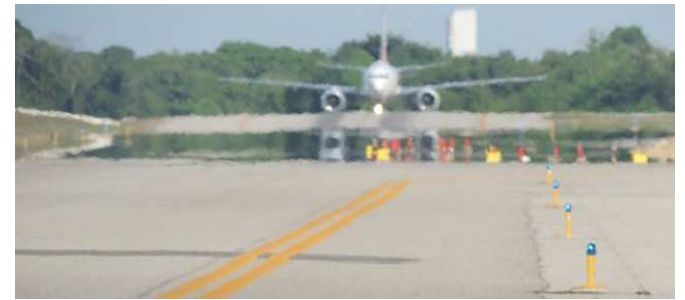

Fig 2

\subsection{Modelagem e Simulação}

Para fundamentar a influencia do alinhamento geométrico no comportamento final, foi utilizado um modelo matemático. A simulação foi desenvolvida em ambiente computacional através do Software LMS Virtual Lab Motion, capaz de desenvolver rapidamente simulações iterativas, para avaliar o desempenho de múltiplas alternativas de design [4].

No modelo temos as entradas correspondentes a cada posição dos trens de pouso, combinados a variáveis intrínsecas ao projeto da aeronave.

\section{Resultados}

Experimento realizado com base em uma aeronave de médio porte. Considerando um total de 40 simulações, alterando os valores nominais de cada uma das 10 posições determinadas de assimetria e paralelismo dos trens de pouso em $25 \%, 50 \%, 70 \%$ e $100 \%$ nas suas tolerâncias especificadas.

Na simulação é verificado o deslocamento da aeronave a uma trajetória de 500 metros na pista.

A tabela 1 mostra a coleta de dados da simulação obtida.

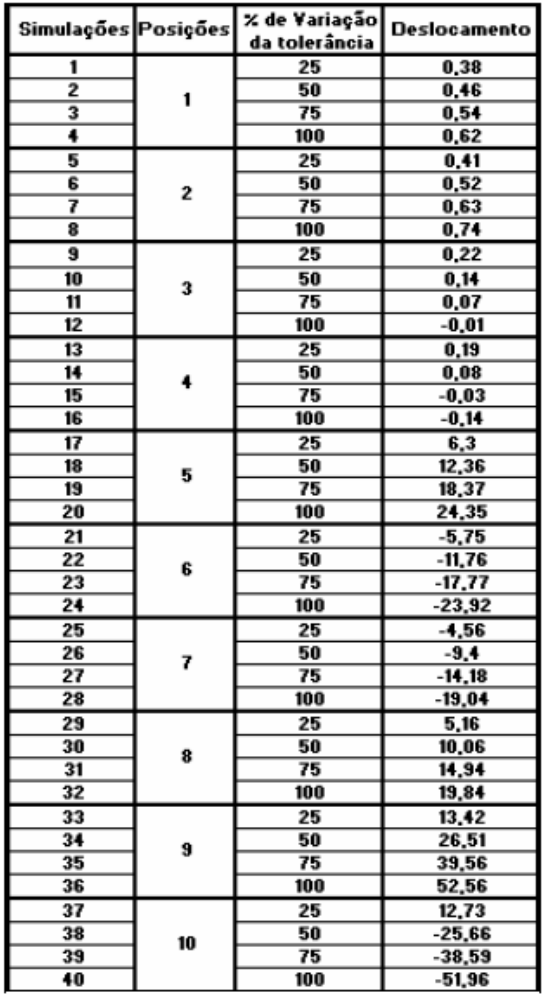

TAB. 1

Transformando os dados da tabela $1 \mathrm{em}$ um arranjo geral para um projeto fatorial 2 fatores, temos a tabela 2. Fazendo o mesmo para as tabelas 3 e 4 (referentes ao paralelismo).

\begin{tabular}{|c|c|c|c|c|}
\hline \multirow{2}{*}{ Posições } & \multicolumn{4}{|c|}{ Variação da Tolerância } \\
\cline { 2 - 5 } & 25 & 50 & 75 & 100 \\
\hline 1 & 0,38 & 0,46 & 0,54 & 0,62 \\
\hline 2 & 0,41 & 0,52 & 0,63 & 0,74 \\
\hline 3 & 0,22 & 0,14 & 0,07 & $-0,01$ \\
\hline 4 & 0,19 & 0,08 & $-0,03$ & $-0,14$ \\
\hline 5 & 6,3 & 12,36 & 18,37 & 24,35 \\
\hline 6 & $-5,75$ & $-11,76$ & $-17,77$ & $-23,92$ \\
\hline 7 & $-4,56$ & $-9,4$ & $-14,18$ & $-19,04$ \\
\hline 8 & 5,16 & 10,06 & 14,94 & 19,84 \\
\hline 9 & 13,42 & 26,51 & 39,56 & 52,56 \\
\hline 10 & 12,73 & $-25,66$ & $-38,59$ & $-51,96$ \\
\hline \multicolumn{5}{|c}{}
\end{tabular}

TAB. 2 


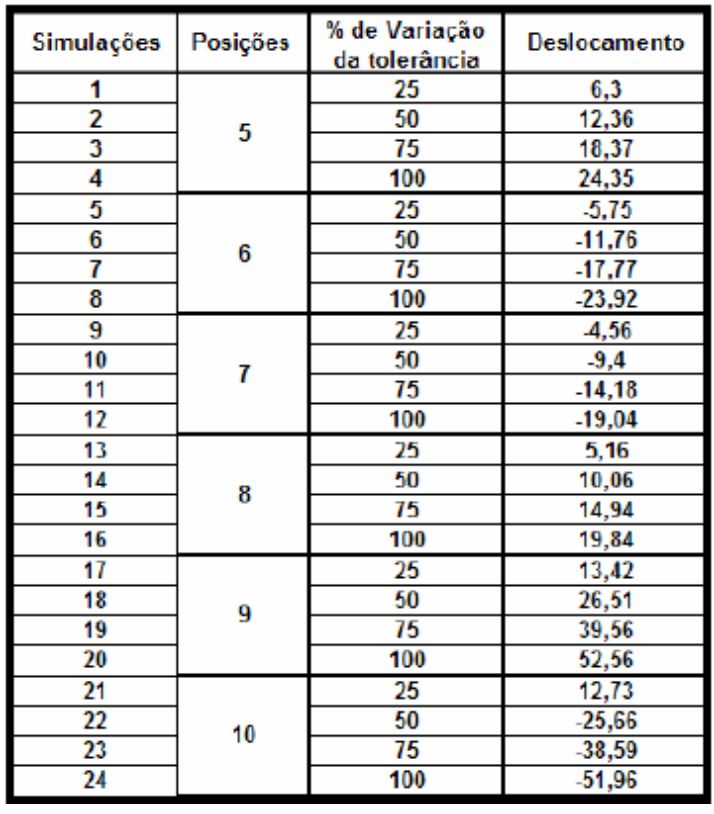

TAB. 3

\begin{tabular}{|c|c|c|c|c|}
\hline \multirow{2}{*}{ Posições } & \multicolumn{4}{|c|}{ Variação da Tolerância } \\
\cline { 2 - 5 } & 25 & 50 & 75 & 100 \\
\hline 5 & 6,3 & 12,36 & 18,37 & 24,35 \\
\hline 6 & $-5,75$ & $-11,76$ & $-17,77$ & $-23,92$ \\
\hline 7 & $-4,56$ & $-9,4$ & $-14,18$ & $-19,04$ \\
\hline 8 & 5,16 & 10,06 & 14,94 & 19,84 \\
\hline 9 & 13,42 & 26,51 & 39,56 & 52,56 \\
\hline 10 & 12,73 & $-25,66$ & $-38,59$ & $-51,96$ \\
\hline
\end{tabular}

TAB. 4

\subsection{Fatores e Níveis}

- A: Posições: 5,6,7,8,9,10 - B: Variação de tolerância: $25,50,75$ e $100 \%$

\subsection{O Modelo estatístico linear}

$\gamma_{\mathrm{ijk}}=\mu+\tau_{\mathrm{i}}+\beta_{\mathrm{j}}+(\tau \beta)_{\mathrm{ij}}+\varepsilon_{\mathrm{ijk}} \quad$ Sendo $\mathrm{i}=1,2 \ldots 10(\mathrm{a}), \mathrm{j}=1,2 \ldots . .4(\mathrm{~b}), \mathrm{k}=1,(\mathrm{n})$ $\mu=$ média global, $\tau=$ Posições do Trem de Pouso, $\beta=$ Variação da tolerância, $\tau \beta=$ Interação Posições $x$ Tolerância

\subsection{Gráfico de Box}

Utilizando o gráfico Box Plot para os fatores da tabela 2, permite-se visualizar de forma rápida e concisa duas situações do comportamento.

O gráfico da figura 3 demonstra a concentração da variação e deslocamento proveniente de cada posição (Fator A), direcionando e permitindo uma análise posterior mais apurada da amplitude do deslocamento de cada ponto. Sendo o cenário de maior concentração e variação, que iremos explorar no decorrer deste estudo (gráficos correspondentes fig 5 e 6.)

Já o gráfico da figura 4, expressa bem o aumento do deslocamento da aeronave mediante a variação da tolerância de cada posição (Fator B).

Estes gráficos podem serem utilizados juntamente com outros gráficos, deixando assim o cenário do experimento mais claro, resultando também em uma análise ainda mais robusta.

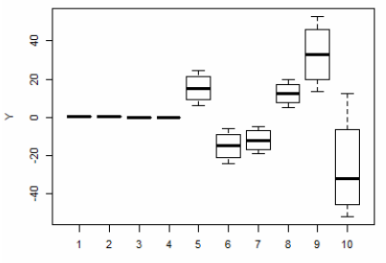

Fig 3

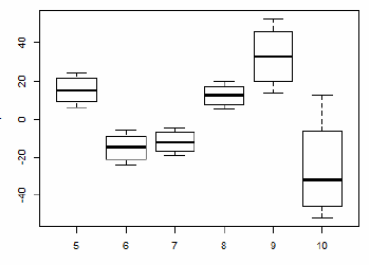

Fig 5

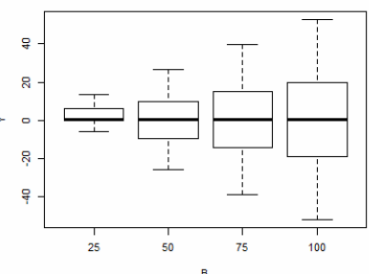

Fig 4

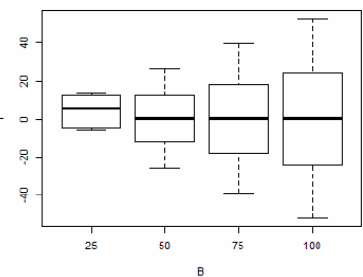

Fig 6

\section{A NOVA}

Com a utilização dos dados do experimento simulado, pretende-se demonstrar a significância dos fatores de posição e se a variação da sua tolerância tem influencia no deslocamento da aeronave.

Através da formula aplicada no $\mathrm{R}$ $A 1=\operatorname{aov}(Y \sim A * B) ; \operatorname{summary}(A 1)$

\section{Df Sum Sq Mean Sq \\ A $\quad 5 \quad 9989 \quad 1997.8$ \\ $\begin{array}{llll}\text { B } & 3 & 79 & 26.5\end{array}$ \\ A:B $15 \quad 3704 \quad 246.9$}

A análise inicial ANOVA permite concluir que as posições (A), a variação $(B)$ e a interação $(A * B)$ são mais significativas e influenciam o valor de deslocamento.

\section{Gráfico de Interação}

Pela formula aplicada no $\mathrm{R}$

with(dados,interaction.plot(A, B,Y,type="b")) , with(dados,interaction.plot(B, A,Y,type="b")) temos

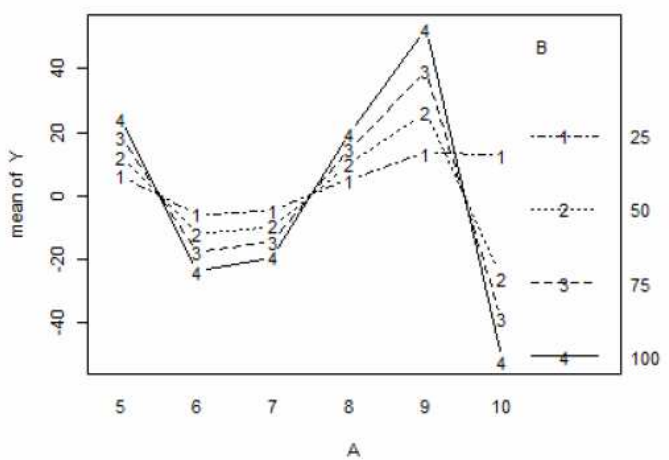

Fig 7 


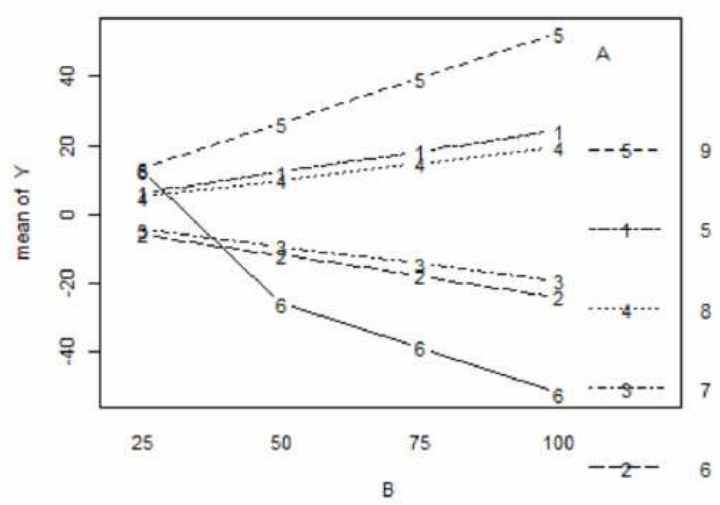

Fig 8

Através do gráfico da figura 7 permite-se identificar interações significantes existentes entre as posições intrínsecas ao fator posição (A), as quais coincidem diretamente com o fenômeno físico. Outro comportamento importante demonstrado de forma direta na figura 8 é a indicação que nas máximas variações das posições 9 e 6 (relacionadas ao trem de pouso dianteiro) que decorrem os maiores deslocamentos da aeronave, servindo de direcionamento para o trabalho de engenheiros de sistemas, projeto e manufatura.

Ajuste do modelo quadrático e análise de variância do modelo estatístico quadrático

A figura 9 apresenta a análise de variância do modelo quadrático abaixo e na figura 10 uma nova análise a partir dos dados significativos. Através da equação abaixo aplicada no $\mathrm{R}$.

$\operatorname{lm} \cdot \operatorname{dados}=\operatorname{lm}\left(\mathrm{Y} \sim \mathrm{A}+\mathrm{B}+\mathrm{A}^{*} \mathrm{~B}+\mathrm{I}\left(\mathrm{A}^{\wedge} 3\right)+\mathrm{I}\left(\mathrm{B}^{\wedge} 3\right)+\mathrm{A}^{*}\right.$

$\mathrm{B}+\mathrm{I}\left(\mathrm{A}^{\wedge} 3^{*} \mathrm{~B}\right)+\mathrm{I}\left(\mathrm{A}^{*} \mathrm{~B}^{\wedge} 3\right)+\mathrm{I}\left(\mathrm{A}^{\wedge} 2\right)+\mathrm{I}\left(\mathrm{B}^{\wedge} 2\right)+\mathrm{A}^{*} \mathrm{~B}+\mathrm{I}\left(\mathrm{A}^{\wedge} 2\right.$

$* \mathrm{~B})+\mathrm{I}\left(\mathrm{A}^{*} \mathrm{~B}^{\wedge} 2\right)$, dados $)$

$>$ summary(lm.dados)

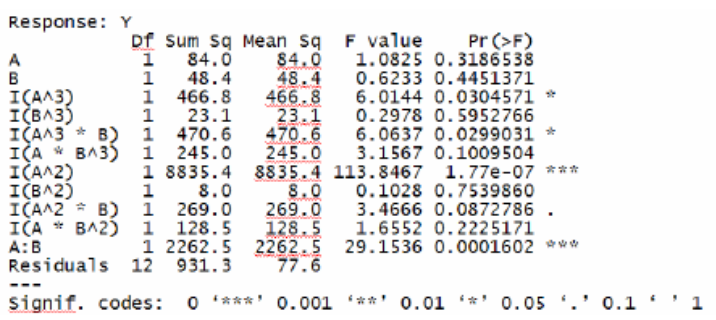

Fig 9

Analysis of Variance Table

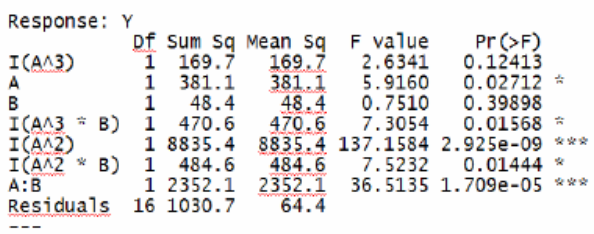

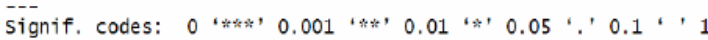

Fig 10

Portanto, podemos confirmar os fatores mais significantes resultantes da análise são: As componentes $\mathrm{A}$ e $\mathrm{I}\left(\mathrm{A}^{\wedge} 2\right)$ representando os efeitos linear e quadrático do fator Posição do Trem de Pouso e os termos $A^{*} B, I\left(A^{\wedge} 3 * B\right)$ e $I\left(A^{\wedge} 2 * B\right)$ são as interações linear e quadrático dos fatores (A) posição do trem de pouso e (B) variação de tolerância.

\section{Análise dos Resíduos}

Através dos gráficos de resíduos, onde demonstra-se que os valores estão todos centrados no zero e com distribuição aparentemente aleatória, permite-se uma avaliação e indicação de boa adequação do modelo proposto.

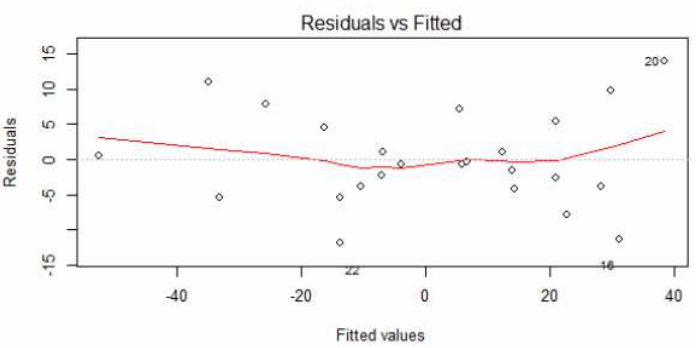

Fig 11

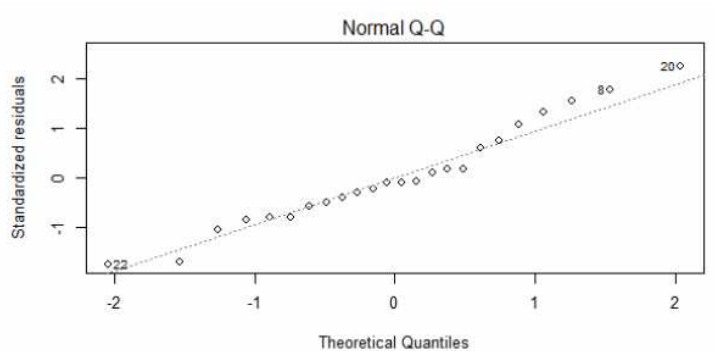

Fig 12

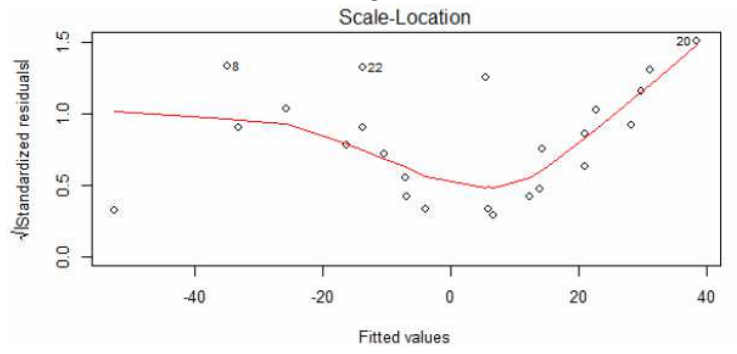

Fig 13

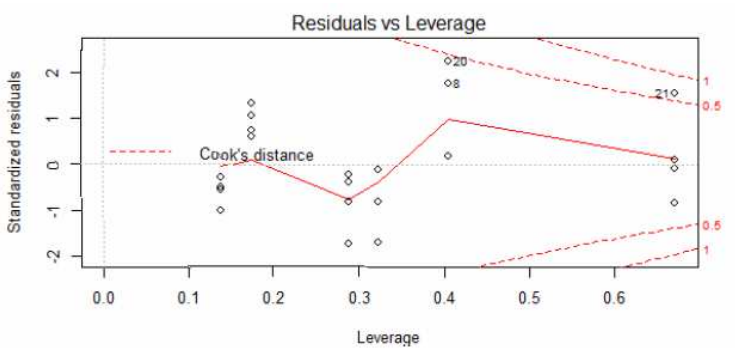

Fig 14

Os gráficos das figuras 15 e 16 (Superfície e Contorno respectivamente) auxiliam na definição que o deslocamento extremo da aeronave é atingido na máxima da variação $(100 \%)$ da tolerância na posição 10 . 

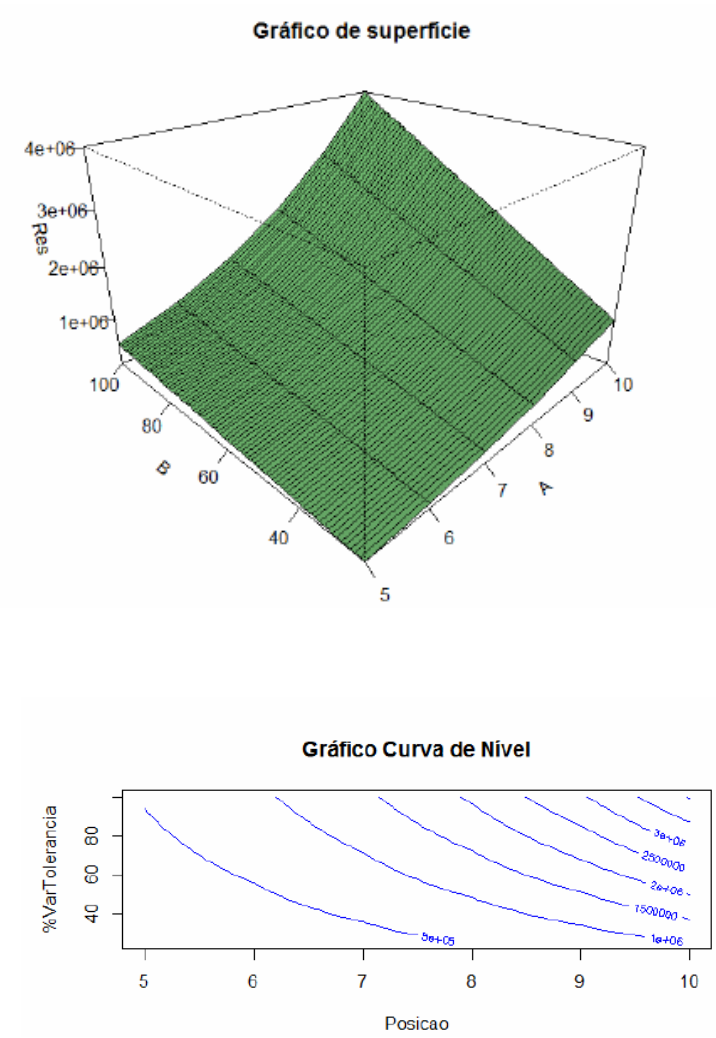

\section{Conclusão}

A análise permitiu destacar de modo estatístico a influência do posicionamento dos trens de pouso e suas variações, complementar a análise do estudo de caso e conhecer ainda mais o fenômeno físico.

Permitiu maior clareza na verificação do comportamento das variáveis envolvidas.

Contribuirá no direcionamento de trabalhos das áreas envolvidas no processo de alinhamento a saber Engenharias de Sistemas, Manufatura e Projeto desde o desenvolvimento do Produto até o processo de fabricação.

Oportunidade de aplicar o mesmo tipo de análise para os demais fatores envolvidos no processo.

Oportunidade de estender a análise para cada fator detalhadamente, utilizando um projeto fatorial $2 \mathrm{k}$.

\section{Contribuição deste artigo}

Os conceitos aplicados neste artigo servirão de base para a elaboração da minha tese de mestrado no Instituto Tecnológico de Aeronáutica saber:

Análise das influências geométricas de uma aeronave na sua trajetória direcional

\section{Referências Bibliográficas}

[1] Regulamentos Brasileiros de Homologação Aeronáutica (RBHA 21.127 - Ensaios - Aeronaves).

[2] Douglas C. Montgomery, Design and Analysis of Experiments, Arizona State University - Fifth Edition

[3] Apostilas do Curso Mecânico de Manutenção de Aeronaves Célula - DAC. CAPÍTULO $2 \mathrm{MON}$ TAGEM E ALINHAMENTO

[4] ww.lmsintl.com/simulation/virtuallab/motion - LMS Engineering Innovation 